Supporting Information

\title{
Antibacterial Activity of Porous Gold Nanocomposites via NIR Light-Triggered Photothermal and Photodynamic Effects
}

Qing Wu, Rui Peng, Yufeng Luo, Qianling Cui, * Shuxian Zhu, Lidong Li*

School of Materials Science and Engineering, University of Science and Technology

Beijing, Beijing 100083, China

Corresponding Author

*E-mail: lidong@mater.ustb.edu.cn (L.L.)

*E-mail: cuiqianling@ustb.ed.cn (Q.C.) 


\section{Experimental Section}

Characterization. Fluorescence emission spectra were recorded using a Hitachi F-7000 fluorescence spectrophotometer. The UV-Vis absorption spectra were measured on a Hitachi U-3900H spectrophotometer. The Zeta-potentials and sizes of the nanoparticles were determined by a Malvern Zetasizer Nano ZS90. The morphologies of the nanoparticles were characterized by a scanning electron microscope (SEM, ZEISS SUPRA55) and transmission electron microscopy (TEM, Hitachi H-7650). Elemental mapping images were captured by TEM equipped with an electron energy spectroscope. Calorimetric measurements were conducted on a MicroCal ITC200. To determine the photothermal conversion, the temperature of solution was measured with an IKA ETS-D5 digital thermometer. MDL-N-808nm-10W laser made by Changchun New Industries Optoelectronics was used as $808 \mathrm{~nm}$ laser source. Power density was $1.5 \mathrm{~W} / \mathrm{cm}^{2}$ and spot area was 78.5 $\mathrm{mm}^{2}$. 

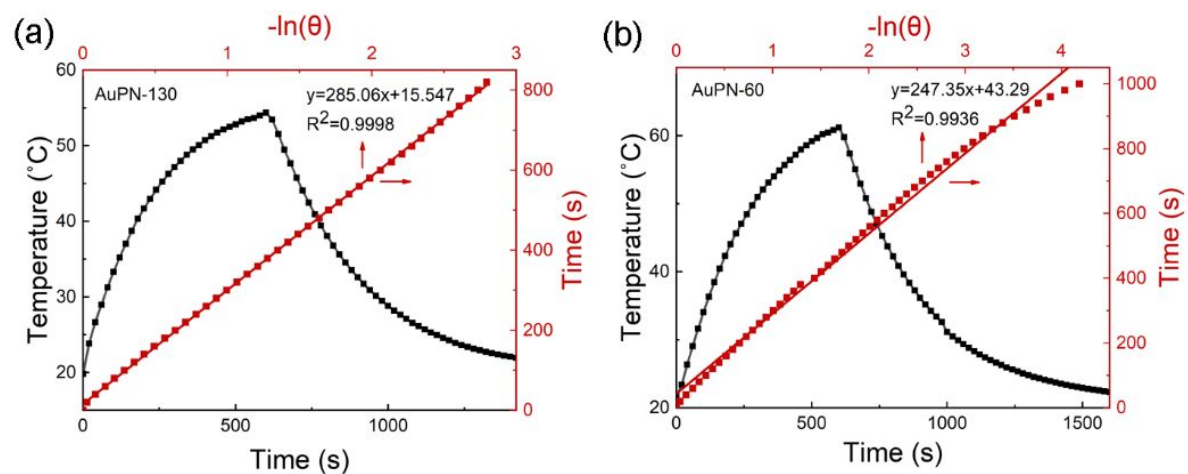

Figure S1. Photothermal conversion efficiency of (a) AuPN-130 and (b) AuPN-60. Temperature variation of AuPNs under the laser on for $600 \mathrm{~s}$ and then off (black dots and line), together with the corresponding time data $v s-\ln (\theta)$ (red dots) and the linear fitting curve (red line).
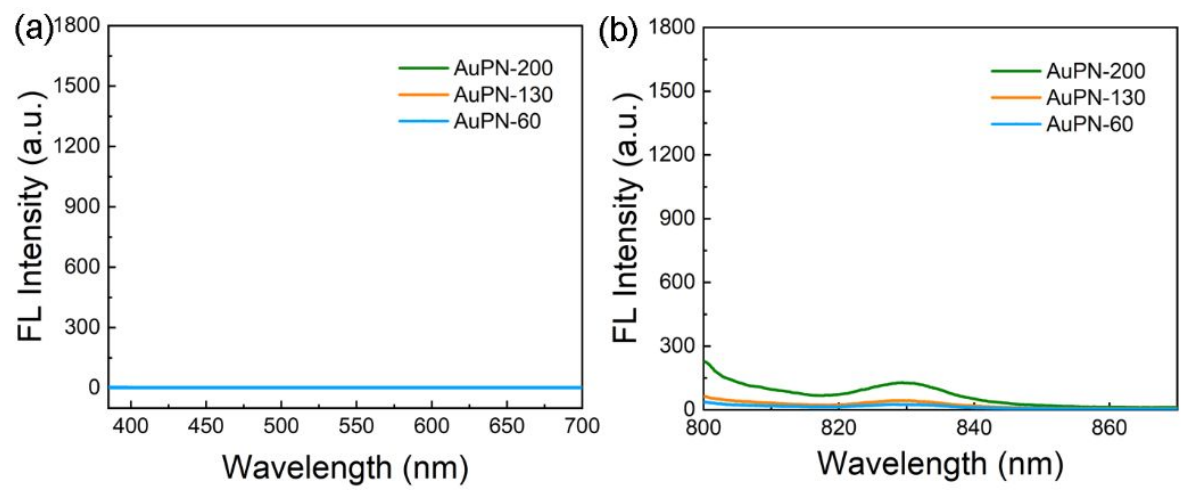

Figure S2. Fluorescence emission spectra of AuPN-200, AuPN-130, AuPN-60 excited by $365 \mathrm{~nm}$ (a) and $780 \mathrm{~nm}$ (b) respectively. The small peak in (b) was caused by its scattering. 


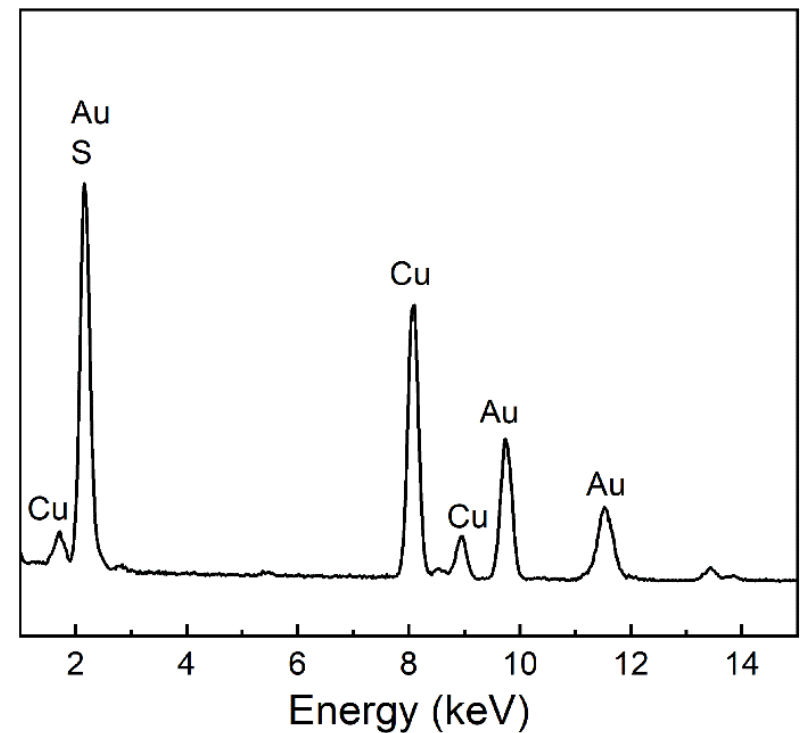

Figure S3. Energy spectrum of AuPN-200@ICG.
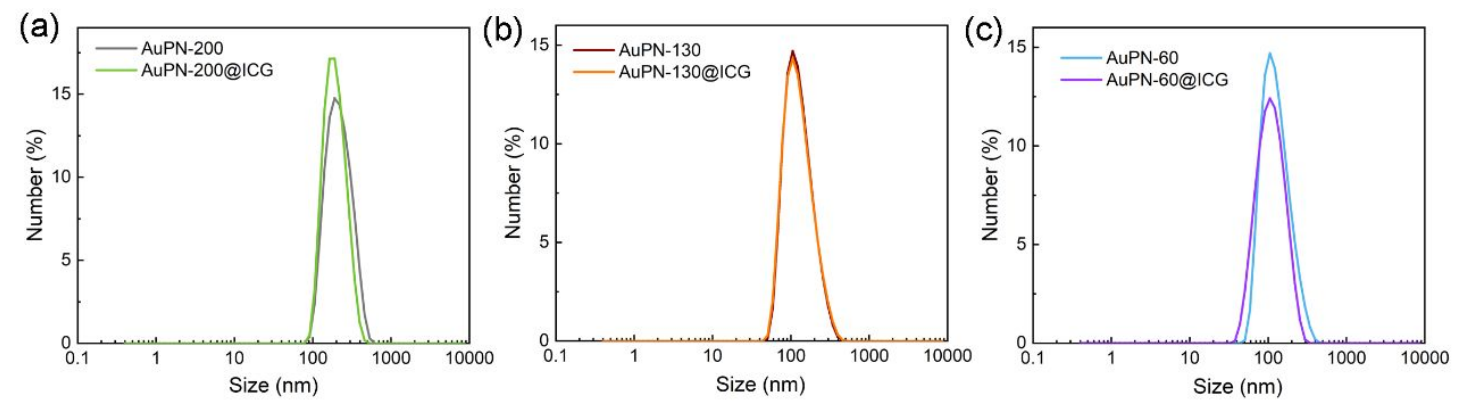

Figure S4. Size distribution determined by DLS of (a) AuPN-200@ICG, (b) AuPN-130@ICG, and (c) AuPN-60@ICG. 

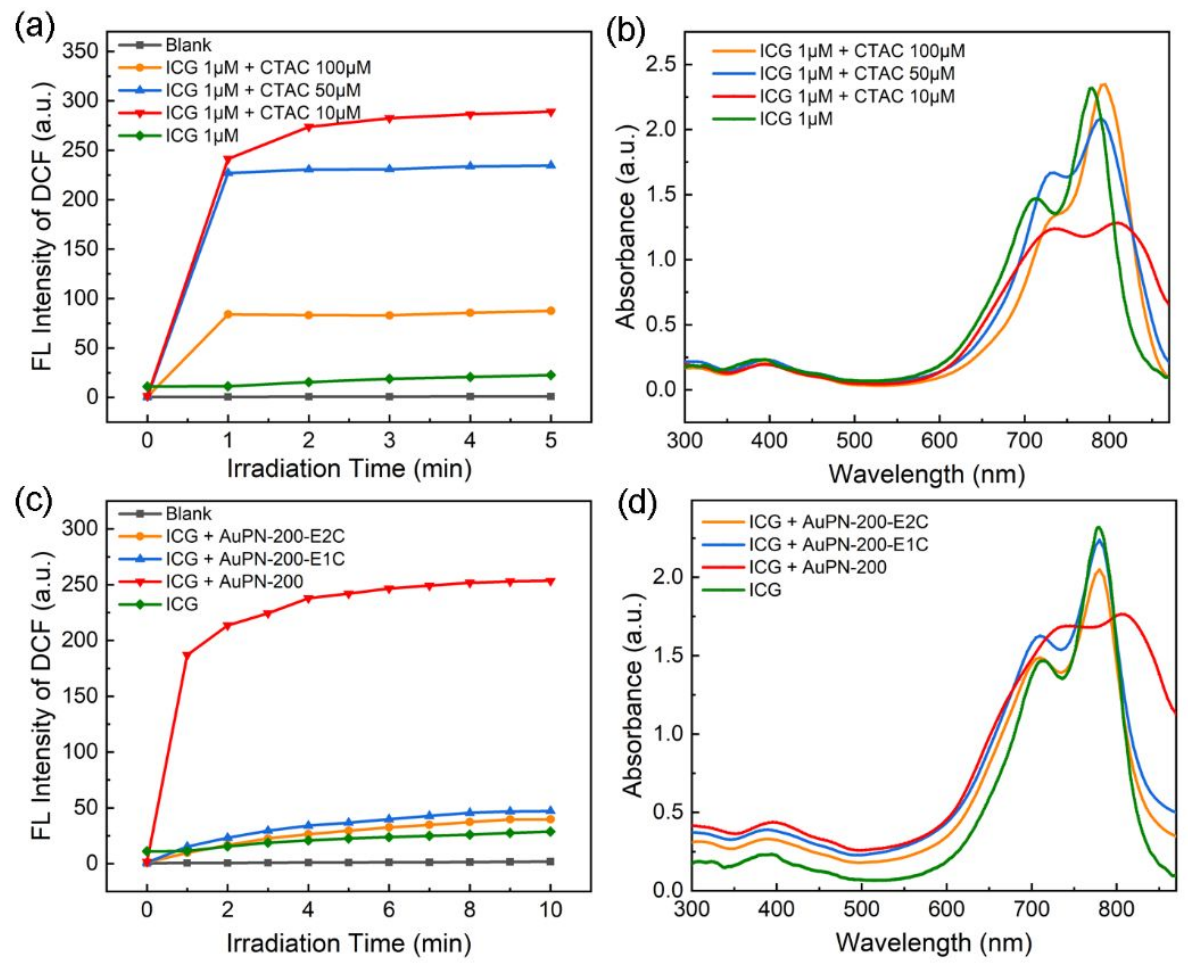

Figure S5. (a) Fluorescence (FL) intensity of DCF at $525 \mathrm{~nm}$ in the presence of ICG and CTAC at different concentration. (b) UV-vis absorption spectra of ICG with CTAC at different concentration. (c) FL intensity of DCF at $525 \mathrm{~nm}$ in the presence of ICG before and after addition of AuPN-200, AuPN-200 with extra once centrifugation (AuPN-200-E1C), AuPN-200 with extra twice centrifugation (AuPN-200-E2C). (d) UV-vis absorption spectra of ICG in the presence of AuPN-200, AuPN-200-E1C, AuPN-200-E2C, respectively. 


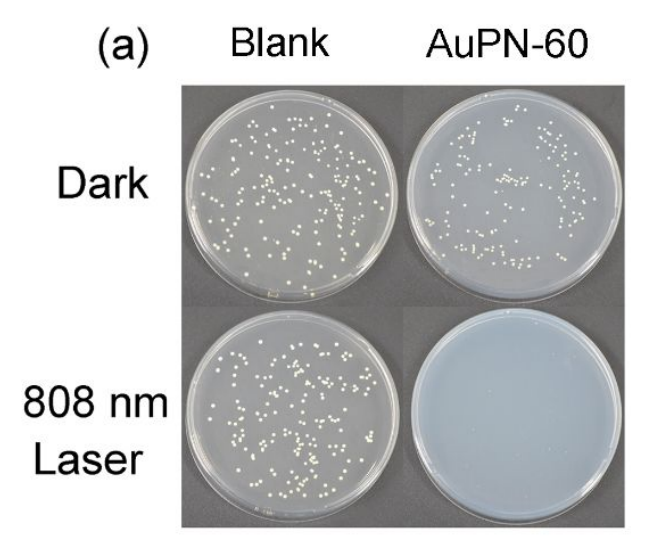

(b)

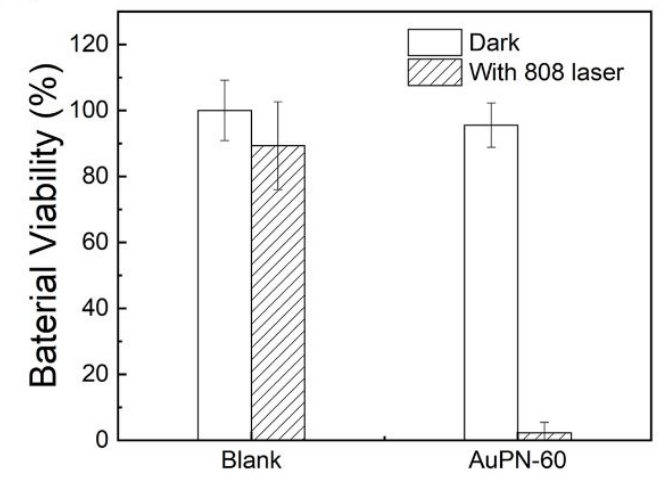

Figure S6. (a) Photographs and (b) bacterial viability of S. aureus colonies grown on the LB agar plate with water, AuPN-60 $(0.33 \mathrm{mg} / \mathrm{mL})$ in dark or under $808 \mathrm{~nm}$ laser irradiation $\left(1.5 \mathrm{~W} / \mathrm{cm}^{2}, 10 \mathrm{~min}\right)$. 